\title{
Crecimiento económico y flujos de efectivo: aplicación al sector industrial de los departamentos de Antioquia y Valle del Cauca (Colombia)***
}

\author{
Economic Growth and Cash Flows: Application to the Industrial \\ Sector in the Departments of Antioquia and Valle del Cauca \\ (Colombia)
}
Crescimento econômico e fluxos de caixa: aplicação para o setor industrial dos departamentos de Antioquia e Valle del Cauca

(Colômbia)

Recibido el 4 de mayo de 2017. Aceptado el 18 de octubre de 2017

\author{
Gabriel Eduardo Escobar Arias*** \\ Colombia
}

Para citar este artículo:

Escobar Arias, Gabriel Eduardo

(junio, 2018). Crecimiento económico y flujos de efectivo:

aplicación al sector industrial

de los departamentos de

Antioquia y Valle del Cauca

(Colombia). Ánfora, 25(44), 65-

84. Universidad Autónoma de

Manizales. ISSN 0121-6538.

\section{Resumen}

Objetivos: determinar la relación entre el crecimiento económico y los flujos de efectivo de las empresas del sector industrial de los departamentos Antioquia y Valle del Cauca. Metodología: se partió de los estados financieros que las empresas industriales de Antioquia y Valle del Cauca reportaron a la Superintendencia de Sociedades de 1995 a 2103. El análisis se aplicó a 102 empresas de Antioquia y 65 del

\footnotetext{
* Antioquia: departamento ubicado al noroccidente de Colombia caracterizado por ser uno de los departamentos de mayor auge industrial que ha tenido Colombia en las últimas décadas. Valle del Cauca: departamento ubicado al occidente de Colombia, caracterizado por tener grandes cultivos de caña de azúcar y el mayor productor de azúcar y alcohol carburante en Colombia.

** Este artículo se deriva de la investigación titulada "Crecimiento económico y flujos de efectivo para el sector industrial en, durante el periodo 1995-2013", con el auspicio de la Universidad Autónoma de Manizales y la Universidad Nacional de Colombia, sede Manizales. El proceso investigativo inició en el mes de abril de 2014 y finalizó en mayo de 2016.

*** PhD in industrial engineering and organizations. Economist. Associate Professor at the Autonoma University of Manizales. Associate professor at the National University of Colombia, Manizales. E-mail address: gabrieledo@autonoma.edu.co
} 
Valle, que hicieron este reporte de forma continua. A estas empresas se les calculó los flujos de efectivo de operación y financiación con los cuales se estableció la relación estadística con el crecimiento de la economía regional durante el mismo periodo con datos del Departamento Nacional de Estadística de Colombia. Resultados: la relación estadística entre los flujos de efectivo de operación y el crecimiento de la economía regional aumentó en Antioquia en 0,389 y en el Valle del Cauca en 0,879; se encontró insatisfactorio este resultado para realizar predicciones, pero al sumarle los flujos de efectivo de operación a la financiación la relación estadística aumentó a 0,740 en Antioquia y en el Valle de Cauca a 0,818; dato más preciso para hacer la predicción de los flujos de efectivo. Conclusiones: por la relación directa entre los flujos de efectivo de las empresas del sector industrial de Antioquia y Valle del Cauca y el crecimiento económico regional, se pueden hacer predicciones de los futuros flujos de efectivo para las empresas del sector.

Palabras clave: Crecimiento económico; Flujo de efectivo; Liquidez.

\section{Abstract}

Objective: to determine the relationship between economic growth and cash flows of industrial sector companies in the departments of Antioquia and Valle del Cauca. Methodology: based on the financial statements that the industrial companies of Antioquia and Valle del Cauca reported to the Superintendency of Corporations between 1995 and 2013. The analysis was conducted with 102 companies in Antioquia and 65 in Valle, which made this an ongoing report. The analysis calculated the operating and financing cash flows with which these companies established the statistical relationship between the growth of the regional economy and data, from the same period, from the National Statistics Department of Colombia. Results: the statistical relationship between operating cash flows and growth of the regional economy increased in Antioquia by 0.389 and in Valle del Cauca by 0.879 . This result was found to be unsatisfactory to make predictions, but when operating cash flows were added to financing, the statistical relationship increased to 0.740 in Antioquia, and Valle de Cauca to 0.818; more precise data to make cash flow prediction. Conclusions: due to the direct relationship between the cash flows of industrial sector companies in Antioquia and Valle del Cauca and the regional economic growth, it is possible to make predictions of future cash flows for companies in this sector.

Keywords: Economic growth; Cash flow; Liquidity. 


\section{Resumo}

Objetivos: determinar a relação entre o crescimento econômico e os fluxos de caixa das empresas do setor industrial dos departamentos de Antioquia e Valle del Cauca. Metodologia: partiu-se dos estados financeiros que as empresas industriais de Antioquia e Valle del Cauca reportaram à Superintendência de Empresas de 1995 até 2103.A análise foi aplicada a 102 empresas e a 65 do Valle, que fizeram este relatório continuamente. Foram calculados os fluxos de caixa de operação e financiamento destas empresas e baseado nestes, foi estabelecida a relação estatística com o crescimento da economia regional durante o mesmo período com dados do Departamento Nacional de Estatísticas da Colômbia. Resultados: A relação estatística entre os fluxos de caixa de operação e o crescimento da economia regional cresceu em Antioquia 0.389 e no Valle del Cauca 0,879; este resultado foi encontrado insatisfatório para fazer previsões, mas ao adicionar os fluxos de caixa de operação ao financiamento, a relação estatística aumentou para 0,740 em Antioquia e no Valle del Cauca para 0818; o qual é um dado mais preciso para prever os fluxos de caixa. Conclusões: pela relação direta entre os fluxos de caixa das companhias do setor industrial de Antioquia e Valle del Cauca e o crescimento econômico regional, podem ser feitas previsões de fluxos de caixa futuras para as empresas do setor.

Palavras-chave: Crescimento econômico; Fluxo de caixa; Liquidez. 


\section{Introducción}

En trabajos anteriores (Escobar, 2014) se analizaron los flujos de efectivo de empresas del sector industrial, comercial, servicios y agropecuario, entre 2002 y 2010 de algunas regiones colombianas. En tal estudio se revisaron sus estructuras de operación, financiación e inversión y se determinó su relación con algunas variables macroeconómicas como el nivel de empleo, la inflación, la tasa representativa del mercado, el producto interno bruto y el índice de la bolsa de valores de Colombia durante el mismo período. Algunos hallazgos, demostraron la correlación positiva entre las variables analizadas como el producto interno bruto, el nivel de empleo, la tasa representativa del mercado, entre otras, con los flujos de efectivo.

De acuerdo con lo anterior, y teniendo como base el mencionado estudio, se evidencia un vacío teórico de la relación entre los resultados de los flujos de efectivo y algunas variables macroeconómicas. Por ello, se indagó en profundidad sobre la relación que pueden presentar variables como el crecimiento económico, los flujos de efectivo y su incidencia en estos, de forma que se pueda realizar su predicción para empresas del sector industrial en el departamento de Antioquia y Valle del Cauca, con una muestra de 102 empresas de Antioquia y 65 del Valle.

Ou y Penman (1989), Sloan (1996), Luo (2008) y Loewk (2014), entre otros, presentaron resultados sobre la predicción de los flujos de efectivo para periodos siguientes, a partir de variables como los flujos de efectivo históricos, los estados financieros principales conformados por el balance general y el estado de resultados o situación económica, el capital de trabajo, los ingresos, los costos y otros informes financieros redactados bajo normas internacionales de información financiera (NIIF). Una de sus conclusiones es que para realizar una predicción más exacta de los flujos de efectivo se debe hacer uso de variables adicionales aunque no señalan cuales; sin embargo, ninguno de ellos, trabajó la predicción a través de la relación que pueda haber entre el crecimiento de la economía y los flujos de efectivo históricos.

En este sentido, se evidencia un vacío teórico entre la relación que puedan tener estas dos variables y la manera de conjugarlas para establecer una predicción de los flujos de efectivo a partir de estos resultados. De acuerdo con lo anterior, surgió la siguiente pregunta de investigación: ¿Cuál es la relación entre el crecimiento económico y los flujos de efectivo en las empresas del sector industrial de Antioquia y Valle del Cauca entre los años 1995 y 2013?

Con base en ello se plantearon los siguientes objetivos: en primer lugar, establecer la relación teórica y práctica entre el crecimiento económico y los flujos de efectivo; segundo, establecer la correlación entre el crecimiento de la economía y los flujos de efectivo durante los años 1995-2013 de las empresas del 
sector industrial del departamento de Antioquia y Valle del Cauca y, finalmente, como consecuencia del estudio, plantear un sistema que permita proyectar los flujos de efectivo a partir de la variación que presenta el crecimiento de la economía; es decir, medir el porcentaje de variación que presentarán los flujos de efectivo ante la variación del PIB de la economía regional en la cual se basa el estudio. Esta observación se fundamenta en que la variable independiente es el crecimiento económico y la variable dependiente serán los flujos de efectivo.

Con tales resultados, los empresarios podrán tener información adicional para realizar sus pronósticos respecto a los flujos de efectivo, pues con información interna y externa podrán realizar una planeación financiera más adecuada para prever su capital de trabajo, las inversiones futuras, el pago de dividendos y de obligaciones financieras, entre otras actividades relacionadas con el desembolso o la adquisición de recursos monetarios o liquidez.

Además, se presenta un análisis en el cual se estable la relación que pueden tener algunos hechos económicos con los flujos de efectivo para las regiones y durante el mismo tiempo, de tal forma que se pueda demostrar que la correlación entre las dos variables se puede explicar de forma cuantitativa y cualitativa.

Finalmente, se espera que los empresarios del sector, los gremios y la academia puedan contar con una herramienta adicional para tomar decisiones que apunten al crecimiento económico de sus organizaciones, pues con información más objetiva o científica pueden obtener resultados más certeros respecto a los flujos de efectivo.

\section{Metodología}

Es una investigación de tipo correlacional en la que se estableció el efecto de la variable independiente, -el crecimiento económico-, ya que la variable dependiente representa los flujos de efectivo. Además, la investigación es de carácter longitudinal, puesto que evalúa varios periodos continuos en cada una de las variables: el crecimiento económico y los flujos de efectivo durante el periodo 1995 a 2013.

Para la recolección de la información se utilizó la base de datos (SIREM) que es operada y suministrada de forma gratuita por la Superintendencia de sociedades. Esta base de datos se encuentra en la página Web de la entidad (www.supersociedades.gov.co).

Para determinar el Producto Interno Bruto se tomó la información suministrada por el Banco Mundial, entidad que tiene proyecciones de crecimiento PIB estimadas hasta el año 2017, mientras que para determinar la población se 
estableció el número de empresas del sector industrial de los departamentos de Antioquia y Valle del Cauca (Colombia) que reportaron estados financieros a la Superintendencia de Sociedades durante 1995 y de las cuales 102 empresas cumplieron con este requisito en Antioquia y 65 empresas del Valle.

Sin embargo, para determinar la muestra se tomaron las empresas que reportaron estados financieros a la Superintendencia de Sociedades entre 1995 y 2013 de forma continua. Se decidió de esta manera, en consideración a que en los periodos en los cuales no hay reporte se tendría un dato cero para la elaboración del aplicativo estadístico, aspecto que afectaría los resultados de la correlación estadística. Para los departamentos de Antioquia y Valle del Cauca, objeto del presente estudio, se obtuvo el siguiente número de empresas: Antioquia 102 y Valle del Cauca 65.

Para el logro de los objetivos se operó de la siguiente manera:

- Se contó con los históricos del Producto Interno Bruto (PIB) desde 1995 hasta 2013

- Se calcularon los flujos de efectivo de las empresas estudiadas a partir de los estados financieros: Balance General y Estado de Resultados, datos suministrados por la Superintendencia de Sociedades entra 1995 y 2013

- Se obtuvo la sumatoria de los flujos de efectivo de las 102 empresas del sector industrial de la región de Antioquia y 65 empresas del sector industrial de la región del Valle a las que se le aplicó el modelo estadístico - A través del software estadístico Statistical Package for the Social Sciences (SPSS) se estableció el grado de asociación lineal entre el PIB y los flujos de efectivo (relación), dato que entregó información importante para determinar el modelo econométrico que se utilizó para establecer los flujos de efectivo futuros

- Se realizó una descripción de los hechos económicos ocurridos en cada región entre 1995 y 2013 con el objeto de determinar la relación de éstos con los resultados de los flujos de efectivo.

Para la estimación de los flujos de efectivo futuros para el período, de acuerdo con la estimación del PIB proyectado, se realizó mediante un modelo de regresión lineal de los flujos de efectivo en función del PIB de la siguiente forma:

$$
\text { F.E.E. }=\text { Constante }+(\text { C x C.E. })+\text { Error }^{1}
$$


De donde:

F.E.E. = Flujo de efectivo esperado.

$\mathrm{C}=$ Coeficiente del PIB.

C.E. = Crecimiento económico esperado.

Para la comprobación y la validez de la regresión de los datos y del modelo planteado se realizaron pruebas estadísticas como La T de Student, que consiste en comprobar si "dos grupos difieren entre sí de manera significativa respecto a sus medias" Sampieri, et al (2006). Y para determinar la validez del modelo, el nivel de significancia de la prueba debe ser menor a 0,05 (Creswell, 2005).

Otra prueba que se aplica al modelo de regresión es la de Fisher (1925), que examina la igualdad entre las varianzas poblacionales de dos variables con distribución normal. Para la aceptación de la prueba bajo este parámetro, su nivel de significancia debe ser menor a 0,05 . La tercera prueba es el coeficiente de determinación, que muestra el valor que puede explicar el efecto de la variable independiente en la variable dependiente. En la medida en que esta se acerque más a 1, significa que hay mayor grado de correlación y por eso los datos son más homogéneos para realizar modelos de predicción (Creswell, 2005).

La siguiente prueba estadística para determinar el modelo de regresión lineal es la normalidad, que muestra si los datos de las variables analizadas tienen una distribución normal, en la medida en que su nivel de significancia sea menor a 0,05, indica que los datos son homogéneos; es decir, que están dentro de la distribución normal. Por lo tanto, los datos son confiables para construir modelos de predicción León y Montero (2003).

La última prueba que se utiliza para comprobar la validez del modelo es la homocedasticidad, que consiste en determinar la igualdad que presentan las varianzas de las variables analizadas. Para determinar que la prueba es válida, su resultado debe ser superior al 5\% de acuerdo con la prueba de Aranaz (2002). Se utilizó el modelo de regresión lineal porque determina el efecto de una variable sobre otra Sampieri et al. (2006); de modo que, para el caso del presente trabajo, permite determinar el efecto del crecimiento económico como variable independiente en los flujos de efectivo como variable dependiente.

\section{Resultados}

Se encontró que la principal variable que une los flujos de efectivo y el crecimiento económico es la liquidez que proporciona el sistema financiero, como los flujos de efectivo a las empresas para que estas puedan realizar la 
inversión necesaria y así aporten al crecimiento de la economía. Esto se sustenta en las relaciones entre los agentes económicos, como lo señalan Aceves y Martínez (2013), quienes demostraron que, en un sistema financiero eficiente que le otorga recursos a las empresas a través del crédito, estos recursos son invertidos de manera eficiente para el crecimiento de la economía y, a la vez, estos recursos (liquidez) provienen de los ahorros de las empresas y las familias como se observa en el siguiente gráfico:

Gráfico 1. Relaciones entre los agentes económicos.

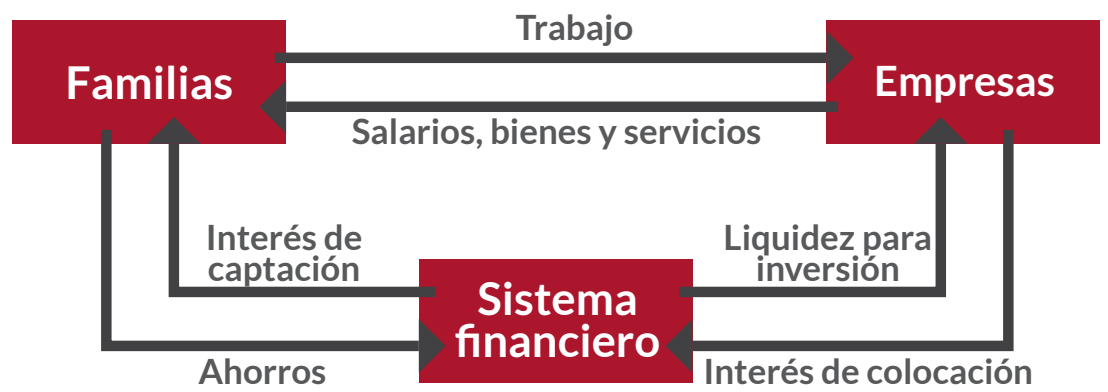

Fuente: elaboración propia.

Según el gráfico, las familias proveen a las empresas el trabajo para que estas puedan producir bienes y servicios que luego serán ofrecidos a las familias para satisfacer sus necesidades; por su parte, las familias reciben un salario por la actividad laboral en las empresas. De este salario (ingreso) las familias destinan una parte al consumo y otra al ahorro, el cual es captado por el sistema financiero que se encarga de suministrar los recursos a las empresas para que realicen sus inversiones. Las familias, por llevar sus ahorros al sistema financiero, reciben una compensación conocida como tasa de interés de captación y las empresas reconocen al sistema financiero una tasa por usar los dineros prestables y que se le conoce como tasa de interés de colocación. La diferencia entre la tasa de interés de captación y de colocación se denomina tasa de intermediación financiera.

De igual modo, el gráfico muestra que el sistema financiero se encarga de entregar la liquidez a las empresas para que estas realicen las inversiones necesarias para producir más y ofrecer más bienes y servicios a la economía; a la vez, a mayor producción, las familias recibirán mayor ingreso y podrán consumir más y ahorrar más. En la medida que esto se dé, como lo indica Solow (1956) en su modelo de crecimiento económico, habrá mayor inversión en capital y, por tanto, mayor crecimiento económico. Es así como las empresas también pueden 
disponer de mayores recursos (flujos de efectivo) mediante la utilidad, dado que, en la medida en que produzcan más y vendan más, dispondrán de una mayor liquidez que podrá ser destinada para el incremento del capital.

El sistema financiero se encarga de proveer parte de la liquidez necesaria que demandan las empresas para realizar sus inversiones; se dice que esta provisión es parcial porque el total de la liquidez proviene de la suma de los créditos y los excedentes de liquidez que las empresas obtienen por su ejercicio comercial. Para que el sistema financiero provea esta liquidez, ofrece varios instrumentos de financiación para que los empresarios, de acuerdo con sus necesidades y requerimientos, puedan escoger las opciones que más se ajusten a sus necesidades y puedan realizar las inversiones necesarias (Levine 1997).

Para proveer estos recursos, el sistema financiero con el tiempo se ha especializado en la formación de diferentes tipos de entidades que colocan los ahorros en recursos para que las empresas puedan realizar sus inversiones; entre ellas, hay algunas como los bancos, las corporaciones financieras, la bolsa de valores, la banca de segundo piso y las fiduciarias.

En este sentido, Terceño y Guercio (2011) demuestran que el desarrollo del sistema financiero interviene en forma directa en el crecimiento económico; es decir, hay una correlación entre el sistema financiero y el crecimiento. El estudio realizado por estos autores fue aplicado en países latinoamericanos, entre ellos Colombia.

De otra parte, en un estudio realizado entre 1990 y 2007 por los mismos autores, donde realizaron una comparación entre el desarrollo del sistema financiero y su incidencia en el crecimiento económico en países como Argentina, Brasil, Chile, Colombia, México, Perú y Venezuela, y encontraron que la correlación entre el desarrollo del sistema financiero y el crecimiento económico es "alta e indiscutible” y la correlación más alta entre estas dos variables se dio en países como Brasil, Colombia, México y Perú.

Para determinar la correlación, los autores utilizaron como variable principal la capitalización del mercado de bonos. Este, como instrumento de financiación, provee liquidez a las empresas para que puedan realizar sus inversiones, aspecto en el cual encontraron que en Colombia la correlación es de 0,90 frente a un valor de uno (1), que es una correlación casi perfecta. Esto indica que en Colombia la inyección de liquidez por el sistema financiero para aportar al crecimiento de la economía es alta a través del mercado de bonos. Esta conclusión se observa también a través de la evolución de operaciones que realiza diariamente la bolsa de valores de Colombia. Según datos de la entidad, desde 2001 hasta la fecha (en 2001 fue creada la BVC en Colombia, producto de la fusión de las bolsas de Medellín, Bogotá y Cali) el mercado de bonos representa el $75 \%$ promedio del total de transacciones realizadas diariamente. 
Otro autor que señala que en Colombia se da el crecimiento económico basado en el mercado financiero es Ruiz (2004), quien mediante un análisis econométrico en el que utiliza como variables el mercado accionario y de bonos, encontró que hay una alta relación estadística entre la liquidez que proveen los instrumentos financieros y el crecimiento de la economía.

En este contexto, en Colombia hay varios tipos de entidades financieras que cumplen su función de intermediación al otorgarle la liquidez necesaria a las empresas para que puedan llevar a cabo sus inversiones y apoyar al crecimiento de la economía, entre ellas:

Tabla 1. Entidades que inyectan liquidez a las empresas y las familias en Colombia

\begin{tabular}{|c|c|c|}
\hline Entidad & Tipo de productos & Colocaciones (clientes) \\
\hline Bancos & $\begin{array}{l}\text { Cuenta de ahorros, cuentas corrien- } \\
\text { tes, banca seguros, crédito de libre } \\
\text { inversión, fomento, tesorería recur- } \\
\text { sos de largo plazo, recursos de corto } \\
\text { plazo, vehículo, vivienda, capital de } \\
\text { trabajo, pymes, leasing, factoring, } \\
\text { descuentos a plazos, cartas de crédi- } \\
\text { to, fiducias, entre otros productos de } \\
\text { colocación y captación. }\end{array}$ & $\begin{array}{l}\text { Personas naturales. } \\
\text { Grandes empresas. } \\
\text { Medianas empresas. } \\
\text { Pequeñas empresas. }\end{array}$ \\
\hline $\begin{array}{c}\text { Corporaciones } \\
\text { financieras }\end{array}$ & $\begin{array}{l}\text { Captación de recursos a través de } \\
\text { Certificados de depósito a término, } \\
\text { colocación de recursos para promo- } \\
\text { ver la industria Colombiana a través } \\
\text { de créditos de largo plazo. }\end{array}$ & $\begin{array}{l}\text { Industria (hoy en Colombia } \\
\text { solamente hay dos entidades de } \\
\text { este tipo pues en la crisis de finales } \\
\text { de los noventa y principios de la } \\
\text { década de dos mil, la mayoría de } \\
\text { estas entidades desaparecieron. } \\
\text { Otro motivo es la poca capacidad } \\
\text { de diversificar productos financie- } \\
\text { ros al realizar sus captaciones y } \\
\text { colocaciones). }\end{array}$ \\
\hline $\begin{array}{l}\text { Compañías de } \\
\text { financiamiento } \\
\text { comercial }\end{array}$ & $\begin{array}{l}\text { Captación de recursos a través de } \\
\text { Certificados de depósito a término y } \\
\text { colocación de recursos para promo- } \\
\text { ver el comercio a través de créditos } \\
\text { de mediano y corto plazo. }\end{array}$ & $\begin{array}{c}\text { Comercializadoras. Entidades que } \\
\text { dejaron de existir en Colombia } \\
\text { por los mismos motivos que se } \\
\text { presentaron para las corporaciones } \\
\text { financieras. }\end{array}$ \\
\hline $\begin{array}{l}\text { Compañías } \\
\text { especializadas } \\
\text { en leasing }\end{array}$ & $\begin{array}{l}\text { Sistema de arrendamiento financiero } \\
\text { utilizado por muchas empresas para } \\
\text { recomponer sus activos de capital. } \\
\text { Hay varios tipos de leasing: financie- } \\
\text { ro, operativo, inmobiliario, sindicado, } \\
\text { de importación, internacional. }\end{array}$ & $\begin{array}{c}\text { Personas naturales. } \\
\text { Grandes, medianas y pequeñas } \\
\text { empresas. }\end{array}$ \\
\hline
\end{tabular}




\begin{tabular}{c|c|c}
\hline Fiduciarias & $\begin{array}{c}\text { Contrato de administración de } \\
\text { recursos monetarios, de activos } \\
\text { inmobiliarios y fideicomisos adminis- } \\
\text { trativos. }\end{array}$ & $\begin{array}{c}\text { Personas naturales. } \\
\text { Personas jurídicas. }\end{array}$ \\
\hline $\begin{array}{c}\text { Bolsa de } \\
\text { valores }\end{array}$ & $\begin{array}{c}\text { Lugar de transacción de activos } \\
\text { financieros de corto y largo plazo } \\
\text { (Acciones, bonos, y divisas.) }\end{array}$ & $\begin{array}{c}\text { Personas naturale colocación de } \\
\text { ción). }\end{array}$ \\
\hline $\begin{array}{c}\text { Fondos de } \\
\text { pensiones y } \\
\text { cesantías. }\end{array}$ & $\begin{array}{c}\text { Captación de recursos para pensión } \\
\text { y cesantía individual. No colocan } \\
\text { recursos a las personas naturales y } \\
\text { jurídicas. }\end{array}$ \\
\hline
\end{tabular}

Fuente: elaboración propia con base en Escobar (2014).

Para demostrar lo expuesto anteriormente, a continuación se presenta, a través de los flujos de caja de financiación, la deuda promedio adquirida por las empresas del sector industrial entre 1995 y 2013 a precios corrientes, recursos que se pueden destinar a diferentes actividades como la inversión. En los departamentos de Antioquia y Valle del Cauca se obtuvieron los siguientes datos:

Tabla 2. Valor deuda promedio adquirida por las empresas en Antioquia y Valle del Cauca, durante los años 1995 - 2013 en miles de pesos.

Departamento / región
Valor de la deuda promedio adquirida durante el periodo 1995 - 2013 en miles de pesos.

\begin{tabular}{cl}
\hline Antioquia & 4.138 .217 \\
\hline Valle del Cauca & 4.164 .774 \\
\hline
\end{tabular}

Fuente: elaboración propia con datos extraídos de la Superintendencia de sociedades (2014).

A través de una correlación estadística se estableció el grado de correlación ante las variaciones del crecimiento económico y los flujos de efectivo operativo para el conjunto de empresas de los departamentos. Para establecer esta correlación se extrajeron los datos del DANE, obteniendo de dicha entidad el PIB por año y luego se calculó la deflactación ${ }^{2}$ a precios de 2008 , con el propósito de igualar a una misma base tanto el valor del PIB como de los flujos de efectivo.

2. Consiste en poner los valores de una serie de tiempo en un mismo periodo o base, con el objeto de analizar los valores de la serie en un periodo de tiempo determinado y poder hacer comparación entre los datos. La base de tiempo la genera el DANE, siendo la última actualización de precios base el año 2008. 
Luego se obtuvo el valor del PIB a precios constantes de 2008; para este proceso, se aplicó la siguiente fórmula:

$\mathrm{PIB}$ a precios $2008=(\mathrm{PIB}$ precios corrientes $/$ deflactor $) \mathrm{x} 100$

Luego, con cada uno de los datos del PIB constantes a precios de 2008 se elaboró el crecimiento económico, que es el resultado de establecer la variación que presentó el PIB constante durante los años comprendidos entre 1995 y 2013. Para determinar esta variación, se utilizó la siguiente fórmula:

Variación PIB = Ln período actual - Ln del año anterior

Las fórmulas para deflactar el PIB y el cálculo de su respectiva variación fueron obtenidas del autor William Sharpe (2003).

Finalmente, para establecer la correlación entre las variables, se obtuvieron de la Superintendencia de Sociedades los flujos de efectivo operativos para cada una de las empresas en cada año. Luego se estableció el valor de dichos flujos de efectivo a precios constantes con base en 2008, para hacer la comparación de los datos en un mismo momento; así, los datos obtenidos por la correlación estadística son más homogéneos, lo que permitió llegar a conclusiones más confiables. En la tabla 3 se muestran los resultados del proceso descrito.

Tabla 3. Crecimiento económico y flujos de efectivo para los departamentos de Antioquia y Valle del Cauca, periodo 1996-2013.

\begin{tabular}{c|c|c|c|c} 
Año & $\begin{array}{c}\text { Crecimiento } \\
\text { económico para } \\
\text { Antioquia durante } \\
\text { los períodos 1996- } \\
2013\end{array}$ & $\begin{array}{c}\text { Flujos de } \\
\text { efectivo deflactados } \\
\text { para Antioquia } \\
\text { durante el período } \\
1995-2013\end{array}$ & $\begin{array}{c}\text { Crecimiento eco- } \\
\text { nómico para Valle } \\
\text { durante el periodo } \\
1996-2013\end{array}$ & $\begin{array}{c}\text { Flujos de efectivo } \\
\text { deflactados para } \\
\text { Valle durante el pe- } \\
\text { riodo 1995-2013 }\end{array}$ \\
\hline 1996 & $-3,29 \%$ & 310.596 .521 & $-1,43 \%$ & 353.774 .826 \\
\hline 1997 & $4,70 \%$ & 380.172 .109 & $0,35 \%$ & 268.226 .711 \\
\hline 1998 & $-4,56 \%$ & 371.503 .792 & $-0,41 \%$ & 302.684 .597 \\
\hline 1999 & $-0,63 \%$ & 366.009 .220 & $-1,61 \%$ & 280.640 .958 \\
\hline 2000 & $0,28 \%$ & 530.955 .705 & $0,22 \%$ & 613.681 .295 \\
\hline 2001 & $-0,05 \%$ & 509.150 .932 & $0,62 \%$ & 600.368 .623 \\
\hline 2002 & $2,05 \%$ & 530.902 .384 & $1,45 \%$ & 512.604 .708 \\
\hline 2003 & $3,02 \%$ & 378.282 .082 & $1,94 \%$ & 559.194 .472 \\
\hline 2004 & $9,35 \%$ & 455.916 .323 & $5,26 \%$ & 672.645 .628 \\
\hline
\end{tabular}




\begin{tabular}{l|l|l|l|l}
\hline 2005 & $4,97 \%$ & 461.389 .936 & $2,05 \%$ & 596.226 .974 \\
\hline 2006 & $7,52 \%$ & 590.258 .460 & $9,10 \%$ & 846.580 .066 \\
\hline 2007 & $6,15 \%$ & 626.432 .326 & $6,98 \%$ & 792.939 .665 \\
\hline 2008 & $-0,46 \%$ & 511.347 .904 & $-0,57 \%$ & 672.407 .337 \\
\hline 2009 & $2,87 \%$ & 412.324 .945 & $4,50 \%$ & 582.516 .657 \\
\hline 2010 & $2,51 \%$ & 471.006 .545 & $1,23 \%$ & 678.940 .737 \\
\hline 2011 & $8,40 \%$ & 452.446 .568 & $3,77 \%$ & 691.535 .311 \\
\hline 2012 & $5,63 \%$ & 396.834 .546 & $4,24 \%$ & 627.831 .968 \\
\hline 2013 & $3,92 \%$ & 521.128 .520 & $4,75 \%$ & 554.856 .077 \\
\hline \multicolumn{7}{|c|}{ Fuente: elaboración propia con datos del DANE (2014). }
\end{tabular}

Para establecer la correlación estadística entre el crecimiento económico y los flujos de efectivo de operación en Antioquia y Valle del Cauca, se partió del crecimiento económico que presentaron entre 1996 y 2013 (tabla 3) y éste se relacionó con los flujos de efectivo deflactados que se encuentran en la tabla; se obtuvo, en el departamento de Antioquia, una correlación de 0,389 y en el departamento del Valle del Cauca 0,879, que constituye un resultado bastante bajo para poder realizar predicciones. Con base en esto se procedió a sumar los flujos de efectivo de operación y financiación para determinar la correlación entre las variables; ello, con el objeto de demostrar que la teoría aplica a la realidad en la medida que, al inyectarle liquidez a las empresas, éstas aprovechan estos recursos para hacer inversiones que apuntan a un mayor crecimiento económico.

Al realizar esta sumatoria entre los flujos de efectivo de operación y financiación y correlacionarla nuevamente con el crecimiento económico, el resultado de la correlación estadística ascendió en Antioquia a 0,740 y en el Valle del Cauca en 0,818; esto demuestra que las variables se pueden utilizar para realizar el análisis de regresión y la posterior predicción de los flujos de efectivo.

Respecto a la correlación estadística entre las variables crecimiento económico y flujos de efectivo de operación y financiación, la homogenización de los datos arroja mejores resultados para demostrar que cuando las empresas generan un mayor flujo se podrán dar mejores resultados de crecimiento en la medida en que los recursos se destinen al crecimiento (Levine 1997). Con la demostración de que los datos son más homogéneos y confiables para realizar la predicción de los flujos, ésta se realizó bajo la siguiente fórmula:

$$
\text { F.E.E. }=\text { Constante }+(\text { C x C.E. })+\text { Error }
$$

Donde F.E.E. es el flujo de efectivo esperado, $\mathrm{C}$ el coeficiente del PIB, C.E. el crecimiento económico esperado. El crecimiento económico esperado se 
obtiene del Banco Mundial y de acuerdo con las consultas realizadas a varios economistas, los datos que presenta dicha institución son confiables; el error que será utilizado como verificador del modelo para determinar si este es funcional estadísticamente con el indicador de normalidad y homocedasticidad, teniendo en cuenta que el error es para cada una de las observaciones de las variables.

De otra parte, según las proyecciones de crecimiento del Banco Mundial para el crecimiento de la economía, se obtuvieron los siguientes datos:

Tabla 4. Proyecciones de crecimiento económico para Colombia para los años 2014 - 2017.

\begin{tabular}{c|c} 
Periodo & $\begin{array}{c}\text { Crecimiento económico } \\
\text { proyectado }\end{array}$ \\
\hline 2014 & $4.7 \%$ \\
\hline 2015 & $4.4 \%$ \\
\hline 2016 & $4.3 \%$ \\
\hline 2017 & $4.3 \%$ \\
\hline
\end{tabular}

Fuente: elaboración propia con datos extraídos del Banco Mundial (2015).

Se observa en esta tabla que las proyecciones del crecimiento económico van disminuyendo año a año, lo que indica que los flujos de efectivo también lo deben hacer de acuerdo con los datos arrojados por la regresión estadística y por la correlación entre el crecimiento económico y los flujos de efectivo. Se establece el crecimiento económico para Colombia puesto que en la búsqueda de la información no hay proyecciones de crecimiento económico para los departamentos analizados. En el proceso de regresión estadística, utilizando las mismas variables y para establecer el modelo adecuado para la predicción de los flujos de efectivo se obtuvieron los siguientes datos:

Tabla 5. Análisis de regresión estadístico para los departamentos de Antioquia y Valle entre los años 1996-2013.

\begin{tabular}{c|c|c} 
Variables estadísticas & Antioquia & Valle del Cauca \\
\hline Constante & 182.696 .086 & 404.471 .757 \\
\hline Coeficiente del PIB & 68.246 .497 & 89.286 .870 \\
\hline
\end{tabular}

Fuente: elaboración propia. 
Con fundamento en el resultado de la tabla anterior, para la predicción de los flujos de efectivo futuros se establece la siguiente fórmula:

$$
\begin{aligned}
& \text { F.E.E. }=182.696 .086+68.246 .497 \times \text { GDP } \\
& \text { F.E.E. }=404.471 .757+89.286 .870 \times \text { GDP }
\end{aligned}
$$

La constante significa que si el valor del PIB de un período es de cero (O\%), es decir, si el crecimiento es nulo, los flujos de efectivo de las empresas del sector industrial en conjunto tendrán un valor para el departamento de Antioquia de \$182.696.086 y para el departamento del Valle \$404.471.757. El coeficiente del PIB significa que ante una variación del crecimiento en un punto equivalente al $100 \%$, los flujos de efectivo aumentarán en \$68.246.497 para Antioquia y para Valle \$89.286.870; también ocurrirá de forma contraria, es decir, si el PIB disminuye en esta cantidad disminuirán los flujos de efectivo futuros. De ahí que determinar el modelo presentado es válido estadísticamente para los departamentos de Antioquia y Valle, por lo que se realizaron las mismas pruebas al modelo que las realizadas para las otras regiones con los siguientes datos:

Tabla 6. Pruebas estadísticas del modelo de regresión bivariado para los departamentos de Antioquia y Valle.

\begin{tabular}{c|c|c} 
Prueba & Resultado de la prueba & Aceptación de la prueba \\
\hline Tde Student: & 4.404 & $\times$ \\
Antioquia & 5.689 & \\
Valle del Cauca & 19.392 & $\times$ \\
\hline Fisher: & 32.367 & \\
Antioquia & & \\
Valle del Cauca & $54.8 \%$ & \\
\hline Coeficiente de & $66.9 \%$ & $\times$ \\
determinación: & & \\
Antioquia & 0.977 & \\
Valle del Cauca & 0.932 & $\times$ \\
\hline Normalidad: & $39.4 \%$ & \\
Antioquia & $5.5 \%$ & \\
Valle del Cauca & & \\
\hline Homocedasticidad: & & \\
Antioquia & & \\
Valle del Cauca & & \\
\hline
\end{tabular}

Fuente: elaboración propia. 
Según los resultados de la tabla anterior, el modelo es válido para realizar la predicción de los flujos de efectivos futuros ante una variación determinada del PIB, puesto que cumple todas las pruebas estadísticas planteadas.

Para determinar el efecto de la variación económica en los flujos de efectivo se aplica la fórmula del modelo establecido y se prueba con diferentes variaciones en el PIB; para medir el cambio de los flujos de efectivo se ensambló la formula y se realizó una simulación en la que el PIB variaba en un punto porcentual con los siguientes resultados:

Tabla 7. Resultados de la variación de los flujos de efectivo para los departamentos de Antioquia ante cambios en el PIB en un punto porcentual.

\begin{tabular}{c|c|c|c|c|cc} 
Constante & $\begin{array}{c}\text { Coeficiente } \\
\text { del PIB }\end{array}$ & $\begin{array}{c}\text { PIB } \\
\text { inicial }\end{array}$ & $\begin{array}{c}\text { PIB } \\
\text { final }\end{array}$ & $\begin{array}{c}\text { Valor del flujo } \\
\text { de efectivo } \\
\text { con el PIB } \\
\text { inicial }\end{array}$ & $\begin{array}{c}\text { Valor del } \\
\text { flujo de } \\
\text { efectivo con } \\
\text { el PIB final }\end{array}$ & $\begin{array}{c}\text { Variación } \\
\text { en el flujo } \\
\text { de efectivo }\end{array}$ \\
\hline 182.696 .086 & 68.246 .497 & $-5 \%$ & $-4 \%$ & 179.283 .761 & 179.966 .226 & $0.3807 \%$ \\
\hline 182.696 .086 & 68.246 .497 & $-4 \%$ & $-3 \%$ & 179.966 .226 & 180.648 .691 & $0.3792 \%$ \\
\hline 182.696 .086 & 68.246 .497 & $-3 \%$ & $-2 \%$ & 180.648 .691 & 181.331 .156 & $0.3778 \%$ \\
\hline 182.696 .086 & 68.246 .497 & $-2 \%$ & $-1 \%$ & 181.331 .156 & 182.013 .621 & $0.3764 \%$ \\
\hline 182.696 .086 & 68.246 .497 & $-1 \%$ & $0 \%$ & 182.013 .621 & 182.696 .086 & $0.3750 \%$ \\
\hline 182.696 .086 & 68.246 .497 & $0 \%$ & $1 \%$ & 182.696 .086 & 183.378 .551 & $0.3736 \%$ \\
\hline 182.696 .086 & 68.246 .497 & $1 \%$ & $2 \%$ & 183.378 .551 & 184.061 .016 & $0.3722 \%$ \\
\hline 182.696 .086 & 68.246 .497 & $2 \%$ & $3 \%$ & 184.061 .016 & 184.743 .481 & $03708 \%$ \\
\hline 182.696 .086 & 68.246 .497 & $3 \%$ & $4 \%$ & 184.743 .481 & 185.425 .946 & $0.3694 \%$ \\
\hline 182.696 .086 & 68.246 .497 & $4 \%$ & $5 \%$ & 185.425 .946 & 186.108 .411 & $0.3681 \%$ \\
\hline 182.696 .086 & 68.246 .497 & $5 \%$ & $6 \%$ & 186.108 .411 & 186.790 .876 & $0.3667 \%$ \\
\hline
\end{tabular}

Fuente: elaboración propia.

Tabla 8. Resultados de la variación de los flujos de efectivo para los departamentos del Valle del Cauca ante cambios en el PIB en un punto porcentual

\begin{tabular}{c|c|c|c|c|cc} 
Constante & $\begin{array}{c}\text { Coeficiente } \\
\text { del PIB }\end{array}$ & $\begin{array}{c}\text { PIB } \\
\text { inicial }\end{array}$ & $\begin{array}{c}\text { PIB } \\
\text { final }\end{array}$ & $\begin{array}{c}\text { Valor del } \\
\text { flujo de efec- } \\
\text { tivo con el } \\
\text { PIB inicial }\end{array}$ & $\begin{array}{c}\text { Valor del } \\
\text { flujo de } \\
\text { efectivo con } \\
\text { el PIB final }\end{array}$ & $\begin{array}{c}\text { Variación } \\
\text { en el flujo } \\
\text { de efectivo }\end{array}$ \\
\hline 404.471 .757 & 89.286 .870 & $-5 \%$ & $-4 \%$ & 400.007 .414 & 400.900 .282 & $0.223 \%$ \\
\hline 404.471 .757 & 89.286 .870 & $-4 \%$ & $-3 \%$ & 400.900 .282 & 401.793 .151 & $0.222 \%$ \\
\hline
\end{tabular}




\begin{tabular}{c|c|c|c|c|cc}
\hline 404.471 .757 & 89.286 .870 & $-3 \%$ & $-2 \%$ & 401.793 .151 & 402.686 .020 & $0.222 \%$ \\
\hline 404.471 .757 & 89.286 .870 & $-2 \%$ & $-1 \%$ & 402.686 .020 & 403.578 .888 & $0.221 \%$ \\
\hline 404.471 .757 & 89.286 .870 & $-1 \%$ & $0 \%$ & 403.578 .888 & 404.471 .757 & $0.221 \%$ \\
\hline 404.471 .757 & 89.286 .870 & $0 \%$ & $1 \%$ & 404.471 .757 & 405.364 .626 & $0.220 \%$ \\
\hline 404.471 .757 & 89.286 .870 & $1 \%$ & $2 \%$ & 405.364 .626 & 406.257 .494 & $0.220 \%$ \\
\hline 404.471 .757 & 89.286 .870 & $2 \%$ & $3 \%$ & 406.257 .494 & 407.150 .363 & $0.219 \%$ \\
\hline 404.471 .757 & 89.286 .870 & $3 \%$ & $4 \%$ & 407.150 .363 & 408.043 .232 & $0.219 \%$ \\
\hline 404.471 .757 & 89.286 .870 & $4 \%$ & $5 \%$ & 408.043 .232 & 408.936 .101 & $0.218 \%$ \\
\hline 404.471 .757 & 89.286 .870 & $5 \%$ & $6 \%$ & 408.936 .101 & 409.828 .969 & $0.218 \%$ \\
\hline
\end{tabular}

Fuente: elaboración propia.

De acuerdo con estos resultados, se comprueba que siempre que el crecimiento económico sea cero por ciento $(0 \%)$ el valor de los flujos de efectivo será igual al valor de la constante y que, por cada punto porcentual que varíe el PIB, los flujos de efectivo variarán en $0,37 \%$ en el departamento de Antioquia y en el Valle en $0,22 \%$ en promedio. Las variaciones en el PIB se realizaron desde $-5 \%$ hasta $6 \%$, pues en esta escala de variación PIB se distribuyen normalmente los datos.

Según esto, si se desea establecer la variación que tendrán los flujos de efectivo del sector industrial de los departamentos de Antioquia y Valle se debe utilizar esta variación; es decir, si el PIB esperado aumenta en un punto porcentual debe incrementar sus flujos de efectivo en $0,37 \%$ y 0,22\% para el próximo período; y si es un decremento en el mismo porcentaje, debe reducir su flujo en $-0,37 \%$ y $-0,22 \%$. También por cada variación en $0,1 \% \mathrm{o}-0,1 \%$ debe incrementar o reducir su flujo en $0,037 \%$ y $-0,037 \%$ y en $0,022 \%$ y -0,022\%, respectivamente.

De esta forma las variaciones en el PIB esperados según los datos del Banco Mundial serán en 2014 del 4,7\% y en 2015 del 4,4\%. Por lo tanto, la variación del PIB presupuestado será de $-0,3 \%$ lo que significa que los flujos de efectivo deben variar para Antioquia en $-0,11 \%(-0,037 \%$ x 3 x 100) y para Valle en $-0,06 \%$ $(-0,022 \%$ x $3 \times 100)$. Y en 2016 el crecimiento en el PIB esperado es del 4,3\%, donde la variación con respecto al año 2015 es de $-0,1 \%$, por lo tanto, los flujos de efectivo deben disminuir para Antioquia en $-0,037 \%(-0,037 \%$ x 1 x 100) y para Valle en -0,022\% (-0,022\% x 1 x 100). Para 2017, no se espera variación en el PIB, por lo que no se generará variación en los flujos de efectivo. Lo anterior se presenta en la siguiente tabla: 
Tabla 9. Predicción de los flujos de efectivo para los años 2014 - 2017 para las empresas del sector industrial de los departamentos de Antioquia y Valle.

\begin{tabular}{c|c|c|c|c|c} 
Periodo & $\begin{array}{c}\text { PIB proyec- } \\
\text { tado }\end{array}$ & $\begin{array}{c}\text { Valor } \\
\text { esperado de } \\
\text { los flujos de } \\
\text { efectivo }\end{array}$ & $\begin{array}{c}\text { Variación } \\
\text { de los flujos } \\
\text { de efectivo }\end{array}$ & $\begin{array}{c}\text { Valor } \\
\text { esperado de } \\
\text { los flujos de } \\
\text { efectivo }\end{array}$ & $\begin{array}{c}\text { Variación de } \\
\text { los flujos de } \\
\text { efectivo }\end{array}$ \\
\hline 2014 & $4.7 \%$ & 185.903 .671 & & 408.668 .240 & \\
\hline 2015 & $4.4 \%$ & 185.698 .932 & $-0.110 \%$ & 408.400 .379 & $-0.066 \%$ \\
\hline 2016 & $4.3 \%$ & 185.630 .685 & $-0.037 \%$ & 408.311 .092 & $-0.022 \%$ \\
\hline 2017 & $4.3 \%$ & 185.630 .685 & $0.000 \%$ & 408.311 .092 & $0.000 \%$ \\
\hline
\end{tabular}

Fuente: elaboración propia.

\section{Conclusiones}

Sloan (1996), Maya (2002) y Gabás (1994) señalan que la predicción de los flujos futuros de efectivo de las empresas se debe realizar a través de variables internas tales como: ingresos históricos, capital de trabajo, precios históricos de las acciones, inventarios, etc. Sin embargo, Ismail y Choi (1996) trataron de explicar los futuros flujos de efectivo a través de la relación que podrían tener estos con algunos factores económicos como la competencia, el tamaño de la empresa y el tipo de producto que esta ofrece, pero ninguno de ellos logró hacer predicciones y encontraron que algunas variables se correlacionan mejor que otras con los flujos de efectivo.

En este contexto, lo propuesto en el presente trabajo no busca determinar que los usos de otras variables no son importantes en la predicción de los flujos de efectivo, pero es preciso tener en cuenta otras como el crecimiento de la economía, donde se encuentra el vacío teórico que se trabajó en este análisis.

La relación entre el crecimiento económico y los flujos de efectivo se determina como la liquidez con la cual cuentan las empresas para destinar recursos a la inversión, que apoyará posteriormente el crecimiento económico; sin embargo, las empresas no tienen en muchos casos los recursos o la liquidez suficiente para llevar a cabo dichas inversiones. Por tanto, recurren al sistema financiero para recoger los recursos adicionales que puedan apalancar la inversión Levine (1997). Por eso, se encontró evidencia empírica respecto a que casi el 100\% de las empresas analizadas recurrieron al crédito financiero para apalancar sus operaciones y sus inversiones. 
En los departamentos de Antioquia y Valle, aproximadamente el 56\% de los años que se analizaron presenta correlación directa entre las variables crecimiento económico y flujos de efectivo. Estas regiones se caracterizan porque el sector cafetero y el sector industrial han sido los protagonistas para la generación del crecimiento de la economía regional. En los últimos años, sin embargo, estos han pasado a un segundo renglón y sectores como la construcción y los servicios están apoyando al crecimiento del PIB. Conviene destacar que las industrias de los departamentos en sus flujos de efectivo dependen de los resultados de las exportaciones y los ingresos que se obtienen a nivel nacional.

Se concluye que en la medida en que los flujos de efectivo reciban mayor liquidez, esta disponibilidad de recursos adicionales mejora la correlación entre las variables. En el desarrollo del presente trabajo se realizó una comparación de correlación entre el crecimiento económico y los flujos de efectivo de operación del sector industrial.

Además, se realizó la correlación entre el crecimiento económico y la sumatoria entre los flujos de efectivo de operación y financiación, llegando a un aproximado del 0.77 , lo que significa que al inyectarle liquidez a las empresas, éstas tienen mayor disponibilidad de recursos que se destinan a la inversión que deriva en crecimiento económico, como lo indica Levine (1997). Esta mayor liquidez la proporcionan los flujos de efectivo de la financiación Terceño y Guercio (2011), que son el resultado de obtener recursos monetarios a través del sistema financiero.

\section{Referencias}

Aceves, S. Martínez, J. (2013). The financial system and his impact in the private sector dynamics. Revista Contaduría y administración, 58, 175-199.

Aranaz, M. (2002). SPSS para Windows. Madrid, España: Mc Graw Hill interamericana.

Banco de la República (2012). Informe de coyuntura económica regional. Retrieved from: http://www.banrep.gov.co/sites/default/files/publicaciones/ archivos/icer_caldas_2012.pdf

Banco Mundial (2015). Real GDP growth at markets prices in percent and current account balance in percent of GDP, unless indicated otherwise. Retrieved from: www.worldbank.org/content/dam/worldbank/GEP/GEP2015a/pdfs/ GEP2015a_chapter2_regionaloutlook_LAC.pdf

Creswell, J. (2005). Educational research: planning, conducting and evaluating quantitative and qualitative research. Pearson Education. 
Departamento Administrativo Nacional de Estadística -DANE. (2014). Estadísticas, producto interno bruto. Retrieved from: www.dane.gov.co/ index. $\quad \mathrm{php} / \mathrm{esp} / \mathrm{pib}-\mathrm{cuentas-nacionales/investigaciones-especiales/77-}$ cuentas- nacionales/cuentas-anuales/

Escobar, G. (2014). Acceso a financiación de las pymes del sector comercio para la ciudad de Manizales. Ánfora, 23(41), 179-201. Manizales, Colombia: Universidad Autónoma de Manizales.

Fisher, R. (1925). Statistical methods for research workers. Oxford, Inglaterrra: Clarendon Press.

Gabás, F. (1994). Capacidad predictiva de los componentes del beneficio: flujos de tesorería y ajustes corto y largo plazo. Revista española de financiación y contabilidad, 24(78), $107-142$.

Ismail, B. Choi, K. (1996). Determinats of time - series propierties of earnings and cash flows. Review of financial economics, 5, 131-145.

León, O. Montero, I. (2003). Métodos de Investigación en psicología y educación. Madrid, España: McGraw-Hill.

Levine, R. (1997). Desarrollo financiero y crecimiento económico: enfoques y temario. Journal of economic literature, 35, 688-726.

Maya, C. (2002). Evidencia empírica sobre la utilidad de la información financiera para la predicción de los resultados futuros. Revista española de financiación y contabilidad, 31(111), 189-224.

Ruiz, A. (2004). Mercados financieros y crecimiento económico en América Latina: un análisis econométrico. Revista análisis económico, 19, 141-165.

Sampieri, R.; Collado, C., Lucio, P. (2006). Metodología de la Investigación. México DF: Mc Graw Hill.

Sloan, R. G. (1996). Do stock process fully reflect information in accruals and cash flows about future earnings? The accounting reviere, 71(3), 289-315.

Solow, R. (1956). A contribution to the theory of economic growth. Quarterly Journal of Economics, 78, 65-94.

Superintendencia de Sociedades. (2014). Sirem, estados financieros sociedades año 1995-2012. Retrieved from: www.supersociedades.gov.co

Terceño, A., Guercio, M. (2011). Economic growth and development of the financial system. A comparative analysis. Investigaciones europeas de dirección y economía de la empresa, 17, 33-46. 